\title{
JUSTIFICACIÓN TOPOLÓGICA DEL ÍNDICE DE HERFINDAHL-HIRSCHMAN COMO ÍNDICE GENERADO POR NORMAS*
}

\author{
DOI: 10.22395/seec.v20n45a5 \\ Carlos Fabián Ruiz Paredes ${ }^{* *}$ \\ Luis Eduardo Ruiz Paredes ${ }^{* *}$ \\ Jorge Morales Paredes ${ }^{* * *}$
}

Recibido: 07 de julio de 2016 • Aprobado: 02 de agosto de 2017

\section{RESUMEN}

Este trabajo propone una manera alterna de justificar el índice de Herfindahl-Hirschman; lo anterior partiendo de índices generados por normas sobre espacios vectoriales de dimensión finita. A partir de normas sobre $\mathbb{R}^{n}$, se pueden construir propuestas para medir concentración industrial. Se probará que el índice de Herfindahl-Hirschman y el índice ratio de concentración $\left(\mathrm{RC}_{1}\right)$ son el límite de los índices generados por la norma euclidiana y la norma del máximo, respectivamente, esto cuando se considera un escenario hipotético de infinitos actores en la industria.

\section{PALABRAS CLAVE}

Índices de concentración; concentración industrial; índice de Herfindahl-Hirschman; Colombia.

\section{CLASIFICACIÓN JEL}

C02, L1 1

\section{CONTENIDOS}

Introducción; 1. Nociones matemáticas y económicas; 2. Desarrollo teórico de los índices; 3. Relaciones entre los índices; 4. Índices cuando se presentan infinitos actores en la industria; 5 . Aplicación, ventajas y análisis económico de los índices generados por normas 6. Conclusiones; Bibliografía.

Este artículo es producto de la investigación "Identificación, planteamiento y análisis de nuevos índices de diversificación de riesgos en las estrategias de inversión basados en el estudio de RDI y HHI", proyecto financiado por la Facultad de Ciencias Económicas y Administrativas de la Universitaria Agustiniana, el cual fue ejecutado entre enero de 2016 y agosto de 2016.

• Economista, Universidad Militar Nueva Granada, Bogotá, Colombia. Magíster en Economía, Universidad Externado de Colombia, Bogotá, Colombia. Candidato a Maestría en investigación de operaciones y estadística, Bogotá, Universidad Tecnológica de Pereira, Colombia. Gerente de proyectos, ProEmprezar SAS, Bogotá, Colombia. Docente de catedra, Universidad Militar Nueva Granada, Bogotá, Colombia. Dirección: Carrera 85 N. ${ }^{\circ}$ 85-50, Apto 301, Bogotá, Colombia. Teléfono móvil 3165878195. Correo electrónico: carlos.ruizp@unimilitar edu.co; fabian_ruiz29@hotmail.com

... Profesional en Finanzas y Comercio Exterior, Fundación Universitaria Empresarial de la Cámara de Comercio de Bogotá, Bogotá, Colombia. Candidato a Maestría en Gestión de Proyectos, EAN, Bogotá, Colombia. Profesor e Investigador de Universitaria Agustiniana, Bogotá, Colombia. Dirección: Av. Ciudad de Cali \#11B-95 Bogotá, Colombia. Teléfono móvil 3105175505. Correo electrónico: luis.ruizp@ uniagustiniana.edu.co

... Matemático, Universidad Nacional de Colombia, Bogotá, Colombia. Maestría en Matemáticas, Universidad Nacional de Colombia, Bogotá, Colombia. Candidato a Doctorado en Matemáticas, Universidad Nacional de Colombia, Bogotá, Colombia. Dirección: Carrera 24 \#1H-45, piso 4, Bogotá, Colombia. Teléfono móvil 3102036877. Correo electrónico: jemoralesp@unal.edu.co 


\title{
TOPOLOGICAL JUSTIFICATION OF THE HERFINDAHL-HIRSCHMAN INDEX AS A NORM-GENERATED INDEX
}

\begin{abstract}
This paper proposes an alternative way of justifying the Herfindahl-Hirschman index; this is based on indices generated by norms on finite dimension vector spaces. Based on $R^{\wedge} n$ standards, proposals to measure industrial concentration can be constructed. The Herfindahl-Hirschman index and the concentration ratio index ( $\mathrm{RCl}$ ) will be proven to be the limit of the indexes generated by the Euclidean norm and the maximum standard, respectively, this when considering a hypothetical scenario of infinite actors in the industry.
\end{abstract}

\section{KEYWORDS}

Concentration index; industrial concentration; Herfindahl-Hirschman index; Colombia.

\section{JEL CLASSIFICATION}

C02, L1 1

\section{CONTENT}

Introduction; 1. Mathematical and economic notions; 2 . Theoretical development of the indices; 3. Relationships between indices; 4 . Indexes when there are infinite actors in the industry; 5. Application, advantages and economic analysis of the indexes generated by standards 6. Conclusions; Bibliography.

\section{JUSTIFICATIVA TOPOLÓGICA DO ÍNDICE DE HERFINDAHL-HIRSCHMAN COMO ÍNDICE GERADO POR NORMAS}

\section{RESUMO}

Este trabalho propõe uma maneira alternativa de justificar o índice de HerfindahlHirschman. Para isso, parte-se de índices gerados por normas sobre espaços vetoriais de dimensão finita. Com base em normas sobre , podem ser construídas propostas para medir a concentração industrial. Prova-se que o índice de Herfindahl-Hirschman e o índice de concentração () são o limite dos índices gerados pela norma euclidiana e pela norma do máximo, respectivamente, isso quando se considera um cenário hipotético de infinitos atores na indústria.

\section{PALAVRAS-CHAVE}

Índices de concentração; concentração industrial; índice de Herfindahl-Hirschman; Colômbia.

\section{CLASSIFICAÇÃO JEL}

C02, L1 1

\section{CONTEÚDO}

Introdução; 1. Noções matemáticas e econômicas; 2. Desenvolvimento teórico dos índices; 3. Relações entre os índices; 4. Índices quando se apresentam infinitos atores na indústria; 5 . Aplicação, vantagens e análise econômica dos índices gerados por normas

6. Conclusões; Bibliografia. 


\section{INTRODUCCIÓN}

El índice de Herfindahl-Hirschman ( $\mathrm{IHH}$ ) es tradicionalmente utilizado por las autoridades de competencia con el fin de calcular y/o medir el grado de concentración de un determinado mercado o una determinada industria, su aplicación se basa en conocer las cuotas de mercado de las firmas y así generar un indicador, el cual permite apreciar el atributo ya mencionado. La literatura ofrece diferentes índices que permiten definir el grado de concentración industrial como en Bikker y Haaf (2002), los cuales requieren conocer el porcentaje de la demanda (cuota de mercado) con el fin de generar el indicador asociado a cada uno de ellos. Esta variedad en los índices sugiere que la información generada por cada uno de ellos es diferente; si bien es cierto todos ellos miden concentración industrial, tambien se reconoce que la información arrojada por las medidas no es la misma, pues la estructura de cada uno de ellos representa un escenario de construcción diferente.

Herfidahl (1950) propone calcular el atributo analizado mediante la sumatoria de las cuotas de mercado elevadas al cuadrado, mientras Davies (1979,p. 67 ) desarrolla el Índice U el cual presenta una estructura diferente a la propuesta del IHH; asimismo, Horvath (1970) propone capturar concentración industrial bajo el índice CCI (Comprehensive industrial concentration index). Lo anterior evidencia que si bien es cierto que las diferentes medidas capturan el grado de concentración industrial, ellas no generan el mismo indicador $\mathrm{y}$, por consiguiente, no muestran la misma información.

La existencia de la variedad en los índices que permiten calcular concentración industrial lleva, sin lugar a duda, a reconocer la posible relación entre dichas mediciones; según Naldi y Flamini (2014) existe correlación entre el IHH y el Ratio de Concentración $\left(\mathrm{RC}_{4}\right)$ bajo diferentes análisis estadísticos; sin embargo, bajo la prueba de preservación de orden, la correlación no es evidente. En terminos generales, es natural afirmar que en efecto la gran variedad de medidas, las cuales tienen como finalidad capturar el grado de concentración de un mercado o una industria, podrían estar relacionadas bajo diferentes criterios.

Con lo anterior, se hace necesario identificar si existe relación entre dichas medidas de concentración con el propósito de justificar el uso de esta amplia variedad de índices o, si por el contrario, es posible llegar a una medida global que muestre la misma información bajo diferentes estructuras de industrias y de mercados. Así, el objetivo de la presente investigación es justificar matemáticamente el IHH y buscar relaciones de este con los índices propuestos en Ruiz (2016) y los índices tradicionalmente usados. Para alcanzar este propósito se realizan las demostraciones matemáticas usando en gran parte cálculo diferencial, así como teoría 
básica de espacios normados y de Banach. Como resultado principal, se prueban las relaciones existentes entre los índices $\mathrm{I}_{2, \infty}, \mathrm{I}_{\alpha, \infty}$ y el $\mathrm{I}_{\infty, \infty}$ con los tradicionalmente usados IHH, Hannah y Kay (HK) y Ratio de Concentración $\left(\mathrm{RC}_{1}\right)$, respectivamente. Dichas relaciones fueron concebidas mediante límites al infinito del número de actores en la industria, por parte de los índices aquí propuestos, adicionalmente, se muestra la ventaja del índice $I_{1}$ generado por la norma del taxista, el cual puede estimar el valor de la concentración industrial reduciendo costos en la recolección de la información.

Este trabajo se desarrolla bajo la siguiente estructura: en primer lugar se hace una breve descripción de espacios normados y algunos de sus resultados más relevantes para uso de la investigación; en segunda instancia se muestran los índices inducidos por normas sobre espacios de dimensión finita como lo realizo Ruiz (2016) y se verifican algunas de sus propiedades deseables a cumplir por los índices que capturan concentración, y, por último, se toman los índices inducidos por normas y se muestra la relación de ellos con los índices tradicionalmente utilizados para medir concentración industrial.

\section{NOCIONES MATEMÁTICAS Y ECONÓMICAS}

\subsection{Una revisión general de los espacios normados}

Recordemos que un espacio vectorial o espacio lineal V sobre un campo K es un conjunto no vacío de elementos $x, y, \ldots$ llamados vectores junto con dos operaciones algebraicas. Estas operaciones son llamadas suma de vectores y multiplicación de vectores por escalares (elementos de K). Si sobre este existe una función llamada norma que es denotada por $\|\cdot\|$ tal que $\|\cdot\|: V \rightarrow \mathbb{R}$ que satisface las siguientes condiciones, cualesquiera que sean $x$, $y \in \mathrm{V}$ y el escalar $\alpha \in \mathrm{K}$.

$$
\begin{gathered}
\|x\| \geq 0, \mathrm{y} \quad\|\mathrm{x}\|=0 \text { si y solamente si } x=0 \\
\|\alpha x\|=|\alpha|\|x\| \\
\|x+y\| \leq\|x\|+\|y\|
\end{gathered}
$$

El espacio es llamado espacio normado, cada norma sobre $\mathrm{V}$ da lugar a una métrica definida por $d(x, y)=\|x-y\|$.

Por ejemplo, si consideramos $\mathrm{V}=\mathbb{R}^{n}$ las siguientes funciones son ejemplos de normas: 


$$
\begin{gathered}
\|x\|_{2}=\sqrt{\sum_{i=1}^{n} x_{i}^{2}} \text { (Norma usual o Euclidea) } \\
\|x\|_{m}=\sum_{i=1}^{n}\left|x_{i}\right| \text { (Norma del taxista o manhattan) } \\
\|x\|_{\infty}=\max _{1 \leq i \leq n}\left|x_{i}\right| \text { (Norma del máximo) } \\
\|x\|_{p}=\left(\sum_{i=1}^{n}\left|x_{i}\right|^{p}\right)^{\frac{1}{p}}, \text { para } p>1 \text {. (Norma lp) }
\end{gathered}
$$

La desigualdad triangular para la norma $\|\cdot\|_{p}$ es conocida como desigualdad de Minkowsky.

La siguiente propiedad de los espacios normados será muy útil a la hora de mostrar la continuidad de los índices propuestos en este trabajo, sean $x, y \in \mathrm{V}:$

$$
\|x\|-\|y\| \leq\|x-y\|
$$

Con las definiciones y propiedades ya mencionadas se pretende empezar con el estudio de un subconjunto del espacio vectorial real $\mathbb{R}^{n}$ sobre el cual se desarrolla la teoría del modelo de Cournot con bienes homogéneos; en la siguiente parte se hace una breve descripción de dicho modelo.

\subsection{Una revisión general del modelo de equilibrio a la Cournot con bienes homogéneos}

En una industria de $n$ firmas se define la cuota $s_{i}$ para la $i$-ésima firma como el porcentaje de la demanda total que le corresponde a dicha firma; el vector formado por las cuotas de todas las firmas de la industria $s=\left(s_{1}, s_{2}, \ldots, s_{n}\right)$ se llamará participación industrial.

El conjunto de todas las participaciones para una industria de firmas es dado por

$$
\mathrm{S}_{n}=\left\{s \in \mathbb{R}^{n}: \sum_{i=1}^{n} s_{i}=1, s_{i} \geq 0, i=1, \ldots, n\right\}
$$

Para los $\operatorname{casos} n=2$ y $n=3$ a continuación: 


\section{Gráfico 1. Conjuntos $S_{2}$ y $S_{3}$}
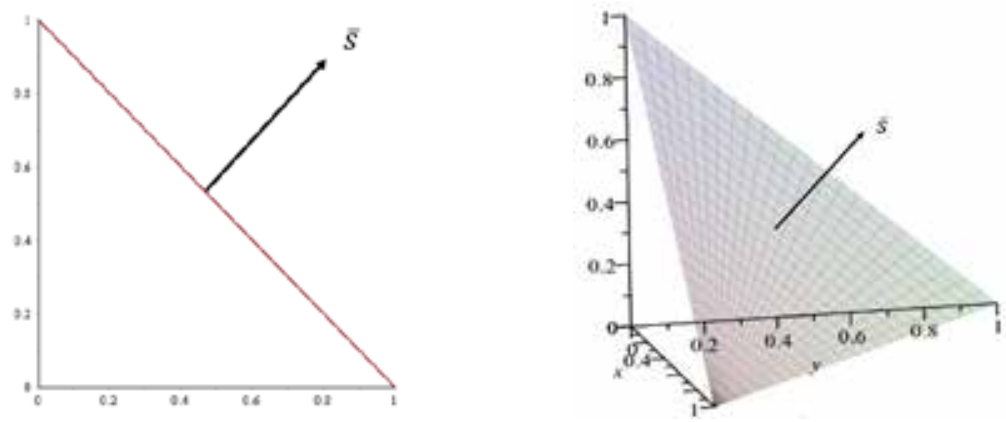

Fuente: elaboración propia

Siguiendo los lineamientos del modelo de competencia de Cournot, como hace referencia Etro (2007), el cual basa su argumento en la competitividad vía cantidades (bajo el supuesto que los costos para cada una de las empresas son los mismos y el bien ofrecido por ella es homogéneo), la búsqueda de equilibrio de Nash-Cournot se fundamenta en encontrar las cantidades ofrecidas por cada una de las firmas (jugadores) competidoras en el mercado; por consiguiente, la función de beneficio para cada una de las firmas viene dada por $\pi_{i}=q_{i}(\mathrm{P}(\mathrm{Q})-c)$ para $i=1, \ldots, n$ donde $n$ es el número de firmas.

Si se supone ahora un modelo en el cual el precio es una función lineal con respecto a $\mathrm{Q}$,

$$
\mathrm{P}(\mathrm{Q})=a-6 \mathrm{Q}
$$

Donde

$$
\mathrm{Q}=\sum_{i=1}^{n} q_{i} \text { y } \quad 6>0
$$

De esta manera el beneficio para la $i$-ésima firma es

$$
\pi_{i}=-b q_{i}^{2}+q_{i}\left(a-b \sum_{\substack{k=1 \\ k \neq i}}^{n} q_{k}-c\right)
$$


Al maximizar esta función se llega a que las cantidades de cada firma son iguales

$$
q_{i}=q_{j} \text { para todo } i, j=1, \ldots, n
$$

Por tanto, la participación del mercado para la firma $i$ debe ser:

$$
s_{i}=\frac{1}{n}
$$

Dado lo anterior, el equilibrio del mercado para el modelo de Cournot con una función inversa de demanda lineal es precisamente el punto $\bar{s}=\left(\frac{1}{n}, \frac{1}{n}, \ldots, \frac{1}{n}\right)$ el cual llamaremos centro del conjunto $\mathrm{S}_{n}$ que corresponde precisamente a una equidistribución de la demanda por parte de las firmas; los casos en los cuales la industria está totalmente concentrada son dados por los elementos de $S_{n}$ que tienen una componente 1 y el resto 0 , es decir, los elementos de $s \in \mathrm{S}_{n}$ tal que $s_{i}=1$ para algún $i=1,2, \ldots, n$.

Diremos que la $i$-ésima firma es dominante si su cuota $s_{i} \geq 1 / n$, y diremos que la $i$-ésima firma es no-dominante si su cuota $s_{i} \leq 1 / n$, si las desigualdades son estrictas diremos estrictamente dominante, y estrictamente no dominante, respectivamente.

Ya con el conjunto $S_{n}$ y su centro definido, en la siguiente sección se desarrollarán los índices inducidos por las normas ya mencionadas sobre $\mathbb{R}^{n}$.

\section{DESARROLLO TEÓRICO DE LOS ÍNDICES}

Esta sección es dedicada a repasar los índices inducidos por métricas, planteados en el trabajo de Ruiz (2016) que sirve de punto de partida para nuestros resultados; se muestran los índices allí construidos y se explican brevemente sus propiedades.

Los índices de concentración industrial buscan medir qué tan alejada se encuentra una industria cualquiera de una industria equitativamente distribuida; sin embargo, en la literatura existen propiedades deseables a cumplir por parte de los índices. En este artículo se listan 6 propiedades tomadas de los axiomas de Hall y Tideman (1967) y Hanna y Kay (1977) que son los más conocidos en la literatura.

Propiedad 1. Independencia de la concentración con respecto al tamaño de la industria, es decir, el índice de concentración es calculado sin importar el tamaño de la industria y dicho tamaño no influye en el indicador. 
Propiedad 2. Simetría significa importar el orden de las cuotas de las firmas a la hora de calcular el índice; este arrojará el mismo dato para todas las permutaciones

Propiedad 3. Continuidad, pequeños cambios en el índice indican pequeños cambios en las participaciones de mercado.

Propiedad 4. Principio de transferencia, si una empresa adquiere más cuota a costo de una empresa más pequeña que ella, el índice aumenta.

Propiedad 5. Normalización, el indicador de concentración debe ser fácil de interpretar; por ende, el rango de la medición es deseable que esté entre cero y uno con el fin de comparar mercado; además, es cero si la industria no presenta concentración, y uno si está completamente concentrada.

Propiedad 6. Fragmentación de firmas, si cada empresa del sector se divide en $k$ partes iguales, la medida de concentración varía en función inversa de $k$.

Como los índices de concentración industrial buscan medir qué tan alejada se encuentra una industria cualquiera de una industria equitativamente distribuida (bajo los supuestos del modelo de competencia a la Cournot), es natural medir la distancia entre una industria $s \in \mathrm{S}_{n}$ y el centro $\bar{s}$.

Sea $\|\cdot\|_{a}$ una norma sobre $\mathbb{R}^{n}$, definimos el índice generado por la norma $\|\cdot\|_{a}$ sobre el conjunto $S_{n}$ como la función:

$$
\begin{gathered}
\mathrm{I}_{a}: \mathrm{S}_{n} \rightarrow \mathbb{R} \\
\mathrm{I}_{a}(s)=\frac{\|s-\bar{s}\|_{a}}{\mathrm{M}_{a}}
\end{gathered}
$$

Donde $\mathrm{M}_{a}$ es el máximo de $\|s-\bar{s}\|_{a}$ sobre $S_{n}$, el cual es alcanzado ya que $S_{n}$ es un conjunto compacto, Apostol (2001), y la norma es una función continua sobre la topología usual de $\mathbb{R}^{n}$, Kreyszig (1989); de esta manera la función $I_{a}$ es continua sobre $S_{n}$ ya que por [8] se tiene

$$
\left|\mathrm{I}_{a}(s)-\mathrm{I}_{a}(r)\right|=\frac{\left\|\left|s-\bar{s}\left\|_{a}-\right\| r-\bar{s} \|_{a}\right|\right.}{\mathrm{M}_{a}} \leq \frac{\|s-r\|_{a}}{\mathrm{M}_{a}}
$$

De esta manera pequeños cambios en el índice indican pequeños cambios en las participaciones de mercado.

Con la construcción anterior de los índices, las propiedades 1 y 3 son satisfechas de manera inmediata por cualquier índice generado a partir de una norma (a 
diferencia de los índices generados por métricas donde la continuidad no se garantiza, por ejemplo, la métrica discreta); las demás propiedades deben verificarse para cada norma.

\section{1 Índice inducido por las normas Ip con $1<\boldsymbol{p}<\infty$}

Tomando la norma lp dada por

$$
\|x\|_{p}=\left(\sum_{i=1}^{n}\left|x_{i}\right|^{p}\right)^{\frac{1}{p}}
$$

Para $1<p<\infty$, se llega al índice $\mathrm{I}_{p}$ :

$$
\mathrm{I}_{p}(s)=n\left(\frac{\sum_{i=1}^{n}\left|s_{i}-\frac{1}{n}\right|^{p}}{(n-1)^{p}+(n-1)}\right)^{\frac{1}{p}}
$$

La propiedad 2 (simetría) es evidente dada la conmutatividad de lsuma.

Para mostrar la propiedad 4 (principio de transferencia), sea $s=\left(s_{1}, \ldots, s_{j}, \ldots\right.$, $\left.s_{k}, \ldots, s_{n}\right)$ una participación industrial dada, se puede suponer gracias a la simetría que las cuotas están organizadas de mayor a menor; de esta manera $s_{j} \geq s_{k}$, sea $\varepsilon<$ 0 la transferencia de cuota de mercado de la firma $k$ hacia la firma $j$, denotemos la nueva participación por:

$$
\hat{s}=\left(s_{1}, \ldots, s_{j}+\varepsilon, \ldots, s_{k}-\varepsilon, \ldots, s_{n}\right)
$$

Definimos la función:

$$
\begin{aligned}
g(\varepsilon) & =\left(\mathrm{I}_{p}(\hat{s})\right)^{p}-\left(\mathrm{I}_{p}(s)\right)^{p} \\
& =\frac{n^{p}}{(n-1)^{p}+(n-1)}\left(\left|s_{j}-\frac{1}{n}+\varepsilon\right|^{p}+\left|s_{k}-\frac{1}{n}-\varepsilon\right|^{p}-\left|s_{j}-\frac{1}{n}\right|^{p}-\left|s_{k}-\frac{1}{n}\right|^{p}\right)
\end{aligned}
$$

Con el fin de analizar el signo de la función $g$ examinamos los siguientes casos:

Caso 1. Si $s_{k}-\frac{1}{n}-\varepsilon \geq 0$, entonces:

$$
g(\varepsilon)=\frac{n^{p}}{(n-1)^{p}+(n-1)}\left[\left(s_{j}-\frac{1}{n}+\varepsilon\right)^{p}+\left(s_{k}-\frac{1}{n}-\varepsilon\right)^{p}-\left|s_{j}-\frac{1}{n}\right|^{p}-\left|s_{k}-\frac{1}{n}\right|^{p}\right]
$$


Derivando con respecto a $\varepsilon$ tenemos:

$$
g^{\prime}(\varepsilon)=\frac{n^{p}}{(n-1)^{p}+(n-1)}\left[p\left(s_{j}-\frac{1}{n}+\varepsilon\right)^{p-1}-p\left(s_{k}-\frac{1}{n}-\varepsilon\right)^{p-1}\right]
$$

Ya que $s_{k}-\frac{1}{n}-\varepsilon<s_{j}-\frac{1}{n}+\varepsilon$, se tiene $g^{\prime}(\varepsilon)>0$.

Caso 2. Si $s_{k}-\frac{1}{n}-\varepsilon<0$.

Caso i. Si $s_{j}-\frac{1}{n}+\varepsilon>0$, entonces:

$$
g(\varepsilon)=\frac{n^{p}}{(n-1)^{p}+(n-1)}\left[\left(s_{j}-\frac{1}{n}+\varepsilon\right)^{p}+\left(\frac{1}{n}+\varepsilon-s_{k}\right)^{p}-\left|s_{j}-\frac{1}{n}\right|^{p}-\left|s_{k}-\frac{1}{n}\right|^{p}\right]
$$

Derivando con respecto a $\varepsilon$ tenemos:

$$
g^{\prime}(\varepsilon)=\frac{n^{p}}{(n-1)^{p}+(n-1)}\left[p\left(s_{j}-\frac{1}{n}+\varepsilon\right)^{p-1}+p\left(\frac{1}{n}+\varepsilon-s_{k}\right)^{p-1}\right]
$$

Ya que por hipótesis $s_{j}-\frac{1}{n}+\varepsilon>0 \mathrm{y} \frac{1}{n}+\varepsilon-s_{k}>0$ se tiene $g^{\prime}(\varepsilon)>0$.

Caso ii. Si $s_{j}-\frac{1}{n}+\varepsilon<0$, así:

$$
(\varepsilon)=\frac{n^{p}}{(n-1)^{p}+(n-1)}\left[\left(\frac{1}{n}-\varepsilon-s_{j}\right)^{p}+\left(\frac{1}{n}+\varepsilon-s_{k}\right)^{p}-\left|s_{j}-\frac{1}{n}\right|^{p}-\left|s_{k}-\frac{1}{n}\right|^{p}\right]
$$

Derivando con respecto a $\varepsilon$ tenemos:

$$
g^{\prime}(\varepsilon)=\frac{n^{p}}{(n-1)^{p}+(n-1)}\left(-p\left(\frac{1}{n}-\varepsilon-s_{j}\right)^{p-1}+p\left(\frac{1}{n}+\varepsilon-s_{k}\right)^{p-1}\right)
$$

Como por hipótesis $s_{j} \geq s_{k}$, se tiene $g^{\prime}(\varepsilon)>0$.

Caso iii. Si $s_{j}-\frac{1}{n}+\varepsilon=0$, así:

$$
g(\varepsilon)=\frac{n^{p}}{(n-1)^{p}+(n-1)}\left[\left(\frac{1}{n}+\varepsilon-s_{k}\right)^{p}-\left|s_{j}-\frac{1}{n}\right|^{p}-\left|s_{k}-\frac{1}{n}\right|^{p}\right]
$$


Derivando con respecto a $\varepsilon$ tenemos:

$$
g^{\prime}(\varepsilon)=\left(p\left(\frac{1}{n}+\varepsilon-s_{k}\right)^{p-1}\right)>0
$$

Lo anterior dado que $\frac{1}{n}+\varepsilon-s_{k}>0$.

De acuerdo con lo anterior $g^{\prime}$ es estrictamente positiva para todo $\varepsilon>0$ y como $g(0)=0$, se tiene que $g(\varepsilon)>0$; así, $\left(\mathrm{I}_{p}(\hat{s})\right)^{p}>\left(\mathrm{I}_{p}(s)\right)^{p}$ tomando raíz $p$-ésima concluimos

$$
I_{p}(\hat{s})>I_{p}(s)
$$

Que es precisamente el principio de transferencia. Como una consecuencia del principio de transferencia y de la simetría se tiene que si $s \in \mathrm{S}_{n}$ con $s$ que no represente una industria totalmente concentrada y $\mu \in S_{n}$ que representa una industria totalmente concentrada, entonces:

$$
\mathrm{I}_{p}(\mu)>\mathrm{I}_{p}(\mathrm{~s})
$$

Si se supone que las cuotas están organizadas de mayor a menor y se puede realizar transferencias totales a la empresa más grande, el máximo $\mathrm{M}_{p}$, mencionado en la construcción del índice, es alcanzado precisamente en las participaciones $\mu$ que representan industrias totalmente concentradas, de lo cual se sigue inmediatamente la propiedad 5 (normalización).

Por último, para la propiedad 6 (fragmentación de firmas), si cada firma del sector se divide en $k$ partes iguales:

Sea $S=\left(s_{1}, s_{2}, \ldots, s_{n}\right)$ la participación para una industria de $n$ firmas y se considera una fragmentación de cada firma en $k$ partes iguales; esto lleva a definir una nueva industria de $n k$ firmas donde la participación de mercado de esta se ilustra en el gráfico 2.

\section{Gráfico 2. Fragmentación de firmas}

$$
s_{f}=(\underbrace{\frac{s_{1}}{k}, \ldots, \frac{s_{1}}{k}}_{k \text {-veces }}, \underbrace{\frac{s_{2}}{k}, \ldots, \frac{s_{2}}{k}}_{k \text {-veces }}, \ldots, \underbrace{\frac{s_{n}}{k}, \ldots, \frac{s_{n}}{k}}_{k \text {-veces }})
$$

Fuente: elaboración propia

Con una verificación directa se ve la siguiente relación entre el índice calculado en esta industria fragmentada y entre la industria original. 


$$
\mathrm{I}_{p}\left(s_{f}\right)=\left(k \frac{(n-1)^{p}+(n-1)}{(n k-1)^{p}+(n k-1)}\right)^{\frac{1}{p}} \mathrm{I}_{p}(s)
$$

Además,

$$
\frac{\partial\left(k \frac{(n-1)^{p}+(n-1)}{(n k-1)^{p}+(n k-1)}\right)^{\frac{1}{p}}}{\partial k}<0
$$

Así la medida de concentración varía en función inversa de k.

\section{2 Índice inducido por la norma del taxista o Manhattan}

Tomando la norma del taxista o Manhattan dada por:

$$
\|x\|_{1}=\sum_{i=1}^{n}\left|x_{i}\right|
$$

Se llega al índice $I_{1}$ :

$$
\mathrm{I}_{1}(s)=n \frac{\sum_{i=1}^{n}\left|s_{i}-\frac{1}{n}\right|}{2 n-2}
$$

Al igual que el índice, la propiedad 2 (simetría) es evidente, dada la conmutatividad de la suma; sin embargo, la propiedad 5 (principio de transferencia) no se cumple; para esto, consideremos, por ejemplo, sea una industria compuesta por 4 firmas cuya participación viene dada por:

$$
s=(0.4,0.3,0.25,0.05)
$$

Si se realiza una transferencia de la firma 2 a la firma 1 de 0.05 , el índice no aumenta ya que se tiene que las nuevas cuotas de mercado son:

$$
s^{*}=(0.45,0.25,0.25,0.05)
$$

y

$$
\mathrm{I}_{1}(s)=\mathrm{I}_{1}\left(s^{*}\right)=0,2 \overline{6}
$$

Donde no aumentó el índice con lo cual no se cumple el principio de transferencia; sin embargo, podemos dar una propiedad más débil la cual llamaremos princi- 
pio de transferencia débil; si una empresa adquiere más cuota, a costo de una empresa más pequeña que ella, el índice aumenta o permanece igual.

Para mostrar este principio de transferencia débil, sea $s=\left(s_{1}, \ldots, s_{j}, \ldots, s_{k}, \ldots\right.$, $s_{n}$ ) una participación industrial dada, organizadas de mayor a menor, sea $\varepsilon>0$ la transferencia de cuota de mercado de la firma $k$ hacia la firma $j$, denotamos la nueva participación por:

$$
\hat{s}=\left(s_{1}, \ldots, s_{j}+\varepsilon, \ldots, s_{k}-\varepsilon, \ldots, s_{n}\right)
$$

Definiendo la función:

$$
f(\varepsilon)=I_{1}(\hat{s})-I_{1}(s)=n \frac{\left|s_{j}-\frac{1}{n}+\varepsilon\right|+\left|s_{k}-\frac{1}{n}-\varepsilon\right|-\left|s_{j}-\frac{1}{n}\right|-\left|s_{k}-\frac{1}{n}\right|}{2 n-2}
$$

Con el fin de analizar el signo de la función $f$ analizamos los siguientes casos:

Caso 1 . Si $s_{j}-\frac{1}{n} \geq 0$, así:

$$
f(\varepsilon)=n \frac{\varepsilon+\left|s_{k}-\frac{1}{n}-\varepsilon\right|-\left|s_{k}-\frac{1}{n}\right|}{2 n-2}
$$

Y ya que $\left|s_{k}-\frac{1}{n}\right| \leq\left|s_{k}-\frac{1}{n}-\varepsilon\right|+\varepsilon$, se tiene $f(\varepsilon) \geq 0$.

Caso 2. Si $s_{j}-\frac{1}{n}<0$

$$
\begin{aligned}
f(\varepsilon) & =n \frac{\left|s_{j}-\frac{1}{n}+\varepsilon\right|-\left(s_{k}-\frac{1}{n}-\varepsilon\right)+\left(s_{j}-\frac{1}{n}\right)+\left(s_{k}-\frac{1}{n}\right)}{2 n-2} \\
& =n \frac{\left|s_{j}-\frac{1}{n}+\varepsilon\right|+s_{j}-\frac{1}{n}+\varepsilon}{2 n-2} \geq 0
\end{aligned}
$$

De los dos casos anteriores se deduce que:

$$
\mathrm{I}_{1}(\hat{s}) \geq \mathrm{I}_{1}(s)
$$

La última desigualdad muestra que se cumple el principio débil de transferencia para $\mathrm{I}_{m}$. Para la propiedad 5 (normalización) solo basta verificar que: 


$$
\mathrm{I}_{1}(\mu) \geq \mathrm{I}_{1}(s)
$$

Y que si $\mathrm{I}_{1}(s)=1$, entonces $s=\mu$ donde $\mu \in \mathrm{S}_{n}$ representa un mercado totalmente concentrado; para esto definamos los conjuntos $\mathrm{D}$ y $\mathrm{D}^{c}$ de la siguiente manera: el primero, el conjunto de subíndices correspondientes a los términos de las firmas dominantes, y el segundo conjunto de subíndices correspondientes a los términos de las firmas estrictamente no-dominantes. Además, se denotara por $|D|$ el cardinal de $\mathrm{D}$.

De esta manera el índice puede ser escrito de la forma:

$$
\mathrm{I}_{1}(s)=\frac{n}{n-2}\left[\sum_{i \in \mathrm{D}}\left(s_{i}-\frac{1}{n}\right)+\sum_{i \in \mathrm{D}^{c}}\left(\frac{1}{n}-s_{i}\right)\right]=\frac{n}{n-2}\left[2 \sum_{i \in \mathrm{D}} s_{i}-\frac{2|\mathrm{D}|}{n}\right]
$$

Para encontrar los puntos donde encuentra el máximo la función $I_{m}$, tomamos el cambio de variables $\Sigma_{\mathrm{i} \in \mathrm{D}} s_{i}=\chi \mathrm{y}|\mathrm{D}|=y$, se tiene:

$$
\mathrm{I}_{1}(x, y)=\frac{n}{n-2}\left[2 x-\frac{2 y}{n}\right]
$$

Donde las variables están restringidas a $\frac{y}{n} \leq x \leq 1,1 \leq y \leq n$

Como $\frac{\partial \mathrm{I}_{1}}{\partial x}>0$ y $\frac{\partial \mathrm{I}_{1}}{\partial y}=<0$, tenemos que la función alcanza su máximo cuando $x=1$ y $y=1$, lo cual implica que el máximo es alcanzado únicamente en las participaciones industriales totalmente concentradas; además, $\mathrm{I}_{1}(\mu)=1$ para toda $\mu \in \mathrm{S}_{n}$ que represente un mercado totalmente concentrado.

Para la propiedad 6 (fragmentación de firmas), sea $s \in S_{n}$ una participación industrial; si cada empresa del sector se divide en $k$ partes iguales, la nueva participación industrial es dada por

$$
s_{f}=\left(\frac{s_{1}}{k}, \ldots, \frac{s_{1}}{k}, \frac{s_{2}}{k}, \ldots, \frac{s_{2}}{k}, \ldots, \frac{s_{n}}{k}, \ldots, \frac{s_{n}}{k}\right)
$$

Y se verifica la relación:

$$
\mathrm{I}_{1}\left(s_{f}\right)=\frac{k(n-1)}{n k-1} \mathrm{I}_{1}(s)
$$


Donde $\frac{\partial\left[\frac{k(n-1)}{n k-1}\right]}{\partial k}<0$, con lo cual la variación del índice (de la industria luego de la fragmentación) está en función decreciente de k.

Un hecho particular acerca del $\mathrm{I}_{1}$ es, sin lugar a duda, la capacidad que tiene este a la hora de capturar la concentración del mercado únicamente usando las cuotas correspondientes a las cuotas de las firmas dominantes del mercado ya que:

$$
\mathrm{I}_{1}(s)=\frac{2 n}{n-2}\left[\sum_{i \in \mathrm{D}} s_{i}-\frac{|\mathrm{D}|}{n}\right]
$$

Esto es una ventaja en la práctica, ya que se requieren menos recursos en la toma de los datos.

Por último, gracias a que cumple el principio débil de transferencia, este índice, además, hace una clara distinción entre las empresas dominantes del mercado y las estrictamente no dominantes; esto, gracias a que si se presenta una transferencia entre empresas dominantes y estas empresas, luego de las transferencias, siguen siendo dominantes, o transferencia entre empresas estrictamente no dominantes, y estas después de las transferencias siguen siendo estrictamente no dominantes, el valor de índice no varía; por el contrario, si la transferencia se realiza entre empresas de diferente tipo (dominantes o no dominantes) o si al realizar la transferencia alguna empresa cambia de dominante a estrictamente no dominante o viceversa, el índice aumenta.

Este índice mide qué tan concentrado está el mercado respecto de las empresas dominantes a las no dominantes, es decir, para este índice lo importante es el valor de la cuota de mercado de las empresas dominantes y el número de estas.

\section{3 Índice inducido por la norma del máximo}

Tomando la norma del máximo dada por:

$$
\|x\|_{\infty}=\max _{1 \leq i \leq n}\left|x_{i}\right|
$$

Se llega al índice $\mathrm{I}_{\propto}$ :

$$
\mathrm{I}_{\infty}(s)=n \frac{\max _{1 \leq i \leq n}\left|s_{i}-\frac{1}{n}\right|}{n-1}
$$


Al igual que el índice anterior, la propiedad 2 (simetría) es evidente, dado que un conjunto no varía al reordenar sus elementos; sin embargo, la propiedad 5 (principio de transferencia) no se cumple; para esto, consideremos por ejemplo, sea una industria compuesta por 4 firmas cuya participación viene dada por $s=(0.4,0.3$, $0.25,0.05)$. Ahora bien, consideremos una transferencia de 0.05 por parte de la firma 3 a la firma 2, las nuevas cuotas de mercado serán $\tilde{s}=(0.4,0.35,0.20,0.05)$ y en este caso el índice no varía, $I_{\infty}(s)=I_{\infty}(\tilde{s})$.

Pero al igual que con el índice generado por la norma del taxista, este índice cumple el principio débil de transferencia, es decir, si una empresa adquiere más cuota a costo de una empresa más pequeña que ella, el índice aumenta o permanece igual.

Para mostrar el principio débil de transferencia, sea $s=\left(s_{1}, s_{2}, \ldots, s_{n}\right)$ la participación para una industria de $n$ firmas, donde las cuotas de mercado están ordenadas de mayor a menorentonces:

$$
\max _{1 \leq i \leq n}\left|s_{i}-\frac{1}{n}\right|=\max \left\{s_{1}-\frac{1}{n}, \frac{1}{n}-s_{n}\right\}
$$

Es decir, este índice es calculado únicamente sobre la empresa con mayor cuota del mercado o la empresa con menor cuota del mercado; de esta manera se evidencia que si se realizan transferencias entre las firmas intermedias (ni la mayor ni la menor) y esta siguen siendo intermedias, el índice no va a variar; en el caso general debemos considerar varios casos; para esto, sea $s=\left(s_{1}, \ldots, s_{k}, \ldots, s_{j}, \ldots, s_{n}\right)$ la participación ordenada de manera decreciente donde $j>k$, si se realiza una transferencia $(\varepsilon)$ de la cuota por parte de la firma $j$ hacia la firma $k$, la nueva participación industrial es

$$
\overleftrightarrow{s}=\left(s_{1}, \ldots, s_{k}+\varepsilon, \ldots, s_{j}-\varepsilon, \ldots, s_{n}\right)
$$

Esta participación no necesariamente está ordenada de manera decreciente; sin embargo, al ordenarla se pueden presentar los siguientes casos:

Caso i. La transferencia cumple las siguientes condiciones $s_{1} \geq s_{k}+\varepsilon$ y $s_{j}-\varepsilon \geq s_{n}$; en este caso el índice permanece igual.

Caso ii. La transferencia cumple las siguientes condiciones $s_{k}+\varepsilon \geq s_{1}$ y $s_{j}-\varepsilon \geq s_{n}$

$$
I_{\infty}(\overleftrightarrow{s})=n \frac{\max \left\{s_{k}+\varepsilon-\frac{1}{n}, \frac{1}{n}-s_{n}\right\}}{n-1}
$$


Dado que max $\left\{s_{1}-\frac{1}{n}, \frac{1}{n}-s_{n}\right\} \leq \max \left\{s_{k}+\varepsilon-\frac{1}{n}, \frac{1}{n}-s_{n}\right\}$, entonces $\mathrm{I}_{\infty}(\overleftrightarrow{s}) \geq \mathrm{I}_{\infty}(s)$.

Caso iii. La transferencia cumple las siguientes condiciones $s_{1} \geq s_{k}+\varepsilon$ y $s_{n} \geq s_{j}-\varepsilon$ Entonces,

$$
\mathrm{I}_{\infty}(\overleftrightarrow{s})=n \frac{\max \left\{s_{1}-\frac{1}{n}, \frac{1}{n}-s_{j}+\varepsilon\right\}}{n-1}
$$

Dado que $\max \left\{s_{1}-\frac{1}{n}, \frac{1}{n}-s_{n}\right\} \leq \max \left\{s_{1}-\frac{1}{n}, \frac{1}{n}-s_{j}+\varepsilon\right\}$, entonces $\mathrm{I}_{\infty}(\overleftrightarrow{s}) \geq \mathrm{I}_{\infty}(s)$.

Caso iv. La transferencia cumple las siguientes condiciones $s_{k}+\varepsilon \geq s_{1}$ y $s_{n} \geq s_{j}-\varepsilon$. Entonces,

$$
\mathrm{I}_{\infty}(\stackrel{\leftrightarrow}{s})=n \frac{\max \left\{s_{k}+\varepsilon-\frac{1}{n}, \frac{1}{n}-s_{j}+\varepsilon\right\}}{n-1}
$$
Dado que $\max \left\{s_{1}-\frac{1}{n}, \frac{1}{n}-s_{n}\right\} \leq \max \left\{s_{k}+\varepsilon-\frac{1}{n}, \frac{1}{n}-s_{j}+\varepsilon\right\}$, entonces
$I_{\infty}(\overleftrightarrow{s}) \geq I_{\infty}(s)$.

Todos los casos anteriores muestran el principio débil de transferencia.

Para la propiedad 5 (normalización) solo basta verificar que

$$
\mathrm{I}_{\infty}(\mu) \geq \mathrm{I}_{\infty}(s)
$$

Y que si $I_{p}(s)=1$, entonces $s=\mu$ donde $\mu \in S_{n}$ representa un mercado totalmente concentrado; para esto debemos encontrar el máximo de

$$
\mathrm{I}_{\infty}(s)=\frac{n \max _{i=1, \ldots, n}\left|s_{i}-\frac{1}{n}\right|}{n-1}
$$

Sobre el conjunto $\mathrm{S}_{n}$, como la función $\mathrm{I}_{\infty}$ es el máximo de un conjunto finito, dicho máximo es alcanzado en algún $k$, para $k \in\{1,2, \ldots, n\}$, fijando dicho $k$, el ejercicio se traduce en maximizar la función

$$
g\left(s_{k}\right)=\left|s_{k}-\frac{1}{n}\right|
$$


Sujeto a $0 \leq s_{k} \leq 1$, así, $0 \leq\left|s_{k}-\frac{1}{n}\right| \leq \frac{n-1}{n}$.

El valor máximo es $\frac{n-1}{n}$ y es alcanzado cuando $\left|s_{k}-\frac{1}{n}\right|=\frac{n-1}{n}$ de lo cual $s_{k}=1$.

Dado que $\sum_{i=1}^{n} s_{i}=1$ entonces $s_{i}=0$ para $i \neq k$. Por consiguiente, el valor máximo que toma la función $I_{\propto}$ es en los puntos que representan mercados totalmente concentrados, además $I_{\propto}(\mu)=1$.

Para la propiedad 6 (fragmentación de firmas), sea $s \in \mathrm{S}_{n}$ una participación industrial; si cada empresa del sector se divide en $k$ partes iguales, la nueva participación industrial es dada por:

$$
s_{f}=\left(\frac{s_{1}}{k}, \ldots, \frac{s_{1}}{k}, \frac{s_{2}}{k}, \ldots, \frac{s_{2}}{k}, \ldots, \frac{s_{n}}{k}, \ldots, \frac{s_{n}}{k}\right)
$$

Y se verifica la relación:

$$
\mathrm{I}_{\infty}\left(s_{f}\right)=\frac{n-1}{n k-1} \mathrm{I}_{\infty}(s)
$$

Donde $\frac{\partial\left[\frac{n-1}{n k-1}\right]}{\partial k}<0$, con lo cual la variación del índice (de la industria luego de la fragmentación) está en función decreciente de $k$.

Nótese que este índice se centra en la empresa cuya cuota está más alejada de la media de las cuotas $1 / n$ (sin tener en cuenta la dominancia o no de dicha firma); además, el índice $I_{\propto}(s)$ para una industria de $n$ firmas significa que la cuota de la firma sobre la cual se calculó está $\mathrm{I}_{\alpha}(s) * 100 \%$ con respecto a la distancia entre $1 / n$ y 1 , alejado de la media de las cuotas; por ejemplo, consideremos una industria de firmas en la cual $I_{\propto}(s)=0.15$, el significado geométrico de esto se ilustra en el gráfico 3.

Así, la firma con participación más alejada de la media de las cuotas, en este caso puede ser la de mayor cuota con 0.32 o la de menor cuota con 0.08 . 


\section{Gráfica 3: Ilustración índice $I_{\infty}$}

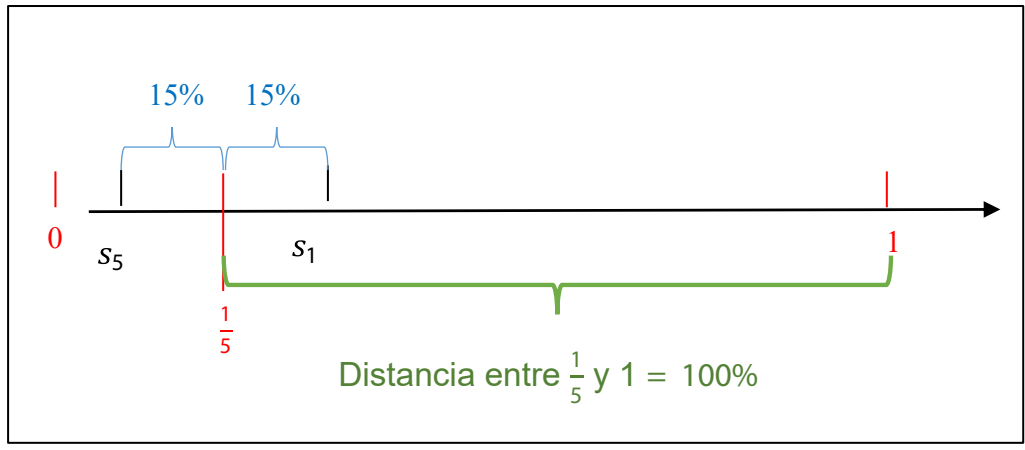

Fuente: elaboración propia

\section{RELACIONES ENTRE LOS ÍNDICES}

Ya con la descripción de los índices generados por normas, en esta sección mostraremos algunas relaciones entre los índices generados por las normas y los índices de Herfindahl-Hirschman y Herfindahl-Hirschman normalizado; estas relaciones se basan en desigualdades ya conocidas para las normas que se aplican a los índices aquí presentados; para esto recordemos la definición de dichos índices, sea $s=\left(s_{1}\right.$, $s_{2}, \ldots, s_{n}$ ) una participación industrial.

$$
\operatorname{IHH}(s)=\sum_{i=1}^{n} s_{i}^{2}
$$

Al normalizar este índice, es decir, al obligar a que su rango sea $[0,1]$ nos encontramos con el Herfindahl-Hirschman Normalizado (IHN) dado por

$$
\operatorname{IHN}(s)=\frac{n \operatorname{lHH}(s)-1}{n-1}
$$

En el caso del índice $I_{2}$ generado por la norma euclídea, sin importar el número de firmas $n$, encontramos una relación cuadrática con el índice Herfindahl-Hirschman Normalizado (y así mismo con el índice de Herfindahl-Hirschman) dada por:

$$
\mathrm{I}_{2}(s)^{2}=\left(\frac{n^{2} \sum_{i=1}^{n}\left(s_{i}-\frac{1}{n}\right)^{2}}{(n-1)^{2}+(n-1)}\right)=n^{2}\left(\frac{\sum_{i=1}^{n} s_{i}^{2}-\frac{2}{n} \sum_{i=1}^{n} s_{i}+\frac{1}{n}}{n^{2}-2 n+1+n-1}\right)=\left(\frac{n \mathrm{HHI}(s)-1}{(n-1)}\right)=\mathrm{IHN}(s)
$$


Otro hecho interesante es que en el caso 2 firmas en la industria $\left(s_{1}+s_{2}=1\right)$ tenemos

$$
\begin{gathered}
I_{1}(s)=\left|s_{1}-\frac{1}{2}\right|+\left|s_{2}-\frac{1}{2}\right|=\left|2 s_{1}-1\right|=\left|s_{1}-s_{2}\right| \\
I_{p}(s)=2\left|\frac{\left|s_{1}-\frac{1}{2}\right|^{p}+\left|s_{2}-\frac{1}{2}\right|^{p}}{2}\right|^{\frac{1}{p}}=\frac{2}{2^{\frac{1}{p}}}|2| s_{1}-\left.\left.\frac{1}{2}\right|^{p}\right|^{\frac{1}{p}}=\left|2 s_{1}-1\right|=\left|s_{1}-s_{2}\right| \\
I_{\infty}(s)=2 \max \left\{\left|s_{1}-\frac{1}{2}\right|,\left|s_{2}-\frac{1}{2}\right|\right\}=2\left|s_{1}-\frac{1}{2}\right|=\left|s_{1}-s_{2}\right|
\end{gathered}
$$

De esta manera todos los índices generados por normas para el caso de una industria con dos firmas miden la diferencia de las cuotas de mercado entre las dos firmas y de esta manera se cumple la igualdad.

$$
\mathrm{I}_{1}(s)=\mathrm{I}_{p}(s)=\mathrm{I}_{\infty}(s)=\sqrt{\operatorname{IHN}(s)}
$$

Para todo $s \in \mathrm{S}_{2}$.

En el caso de una industria de tres firmas no se alcanza a tener la relación anterior; sin embargo, para tres firmas tenemos (por simetría podemos asumir que las cuotas de mercado de las firmas están ordenadas de mayor a menor).

$$
I_{1}(s)=3 \frac{\left|s_{1}-\frac{1}{3}\right|+\left|s_{2}-\frac{1}{3}\right|+\left|s_{3}-\frac{1}{3}\right|}{4}=3 \frac{\left|s_{1}-\frac{1}{3}\right|+\left|s_{1}+s_{3}-\frac{2}{3}\right|+\left|s_{3}-\frac{1}{3}\right|}{4}
$$

Y así,

$$
I_{1}(s)= \begin{cases}\frac{1-3 s_{3}}{2}, & s_{1}+s_{3}-\frac{2}{3}<0 \\ \frac{3 s_{1}-1}{2}, & s_{1}+s_{3}-\frac{2}{3} \geq 0\end{cases}
$$

Por otra parte,

$$
\mathrm{I}_{\infty}(s)=\frac{3}{2} \max \left\{s_{1}-\frac{1}{3}, \frac{1}{3}-s_{3}\right\}
$$


Considerando los dos casos:

$$
I_{\infty}(s)=\left\{\begin{array}{l}
\frac{3}{2}\left(\frac{1}{3}-s_{3}\right), s_{1}+s_{3}-\frac{2}{3}<0 \\
\frac{3}{2}\left(s_{1}-\frac{1}{3}\right), s_{1}+s_{3}-\frac{2}{3} \geq 0
\end{array}\right.
$$

De lo cual:

$$
\mathrm{I}_{1}(\mathrm{~s})=\mathrm{I}_{\infty}(\mathrm{s})
$$

Para todo $s \in \mathrm{S}_{3}$.

En los casos de mayor número de firmas ya no se tiene la igualdad entre índices; sin embargo, como todas las normas sobre $\mathbb{R}^{n}$ son equivalentes y en particular gracias a la relación.

$$
\begin{gathered}
\|x\|_{\infty} \leq\|x\|_{p} \leq n^{\frac{1}{p}}\|x\|_{\infty} \\
\frac{n}{n-1}\|s-\bar{s}\|_{\infty} \leq \frac{n}{n-1} \frac{\left((n-1)^{p}+(n-1)\right)^{\frac{1}{p}}}{\left((n-1)^{p}+(n-1)\right)^{\frac{1}{p}}}\|s-\bar{s}\|_{p} \leq \frac{n^{\frac{1}{p}+1}}{n-1}\|s-\bar{s}\|_{\infty}
\end{gathered}
$$

y así

$$
\mathrm{I}_{\infty}(s) \leq \frac{\left((n-1)^{p}+(n-1)\right)^{\frac{1}{p}}}{n-1} \mathrm{I}_{p}(s) \leq n^{\frac{1}{p}} \mathrm{I}_{\infty}(s)
$$

En el caso del índice $\mathrm{I}_{1}$ se tiene el análogo

$$
\mathrm{I}_{\infty}(s) \leq 2 \mathrm{I}(s) \leq n \mathrm{I}_{\infty}(s)
$$

En particular se encuentra una relación con el índice de Herfindahl-Hirschman Normalizado dada por

$$
\frac{(n-1)}{n} \mathrm{I}_{\infty}(s)^{2} \leq \mathrm{IHN}(s) \leq(n-1) \mathrm{I}_{\infty}(s)^{2}
$$




\section{4. ÍNDICES CUANDO SE PRESENTAN INFINITOS ACTORES EN LA INDUSTRIA}

Los índices mostrados en la sección 2 asumen que el número de firmas presentes en una industria se mantiene estático (es decir, no varía); sin embargo, desde otro punto de vista se puede asumir que el número total de firmas es infinito (ya que en cada momento pueden ir apareciendo firmas nuevas) y la participación del mercado sería dada por la sucesión $s=\left(s_{1}, s_{2}, s_{3}, \ldots\right)$, donde lo que se espera es que la serie:

$$
\sum_{i=1}^{\infty} s_{i}=1
$$

Aunque en la práctica los excepto para un numero finito de elementos.

Es decir, que podemos crear índices para infinitas firmas cambiando el conjunto $\mathrm{S}_{n}$ por un conjunto como

$$
S_{\infty}=\left\{\begin{array}{l}
s=\left(s_{1}, s_{2}, \ldots\right) \mid \sum_{i=1}^{\infty} s_{i}=1, s_{i} \geq 0, s_{i}=0 \\
\text { excepto un número finito de elementos }
\end{array}\right\}
$$

Y por último, tomar el límite de cada índice cuando $n$ tiende a infinito.

Identificando cada elemento de $s \in \mathrm{S}_{n}$ con un elemento de $\mathrm{S}_{\infty}$ por medio de la inmersión natural

$$
\begin{gathered}
j: S_{n} \rightarrow S_{\infty} \\
j\left(s_{1}, s_{2}, \ldots, s_{n}\right)=\left(s_{1}, s_{2}, \ldots, s_{n}, 0,0, \ldots\right)
\end{gathered}
$$

Podemos ver una contenencia $S_{n} \subset S_{\infty}$ para cada $n \in \mathbb{Z}^{+}$. (Note que en realidad tenemos $\bigcup_{n \geq 1} S_{n}$ ).

En el caso para el índice $\mathrm{I}_{p}$, por ejemplo, podemos definir un índice sobre $\mathrm{S}_{\infty}$ como

$$
I_{p, \infty}(s)=\lim _{n \rightarrow \infty} n\left(\frac{\sum_{i=1}^{n}\left|s_{i}-\frac{1}{n}\right|^{p}}{(n-1)^{p}+(n-1)}\right)^{\frac{1}{p}}
$$

Ya que $\lim _{n \rightarrow \infty} \frac{n}{\left((n-1)^{p}+(n-1)\right)^{\frac{1}{p}}}=1$ y como: 


$$
\sum_{i=1}^{n}\left|s_{i}-\frac{1}{n}\right|^{p} \leq \sum_{i=1}^{\infty}\left|s_{i}-\frac{1}{n}\right|^{p}
$$

Para todo $n$, además la serie de la parte derecha converge puesto que existe una constante $c_{p}$ que depende únicamente de $p$ tal que:

$$
\begin{aligned}
& \left|s_{i}-\frac{1}{n}\right|^{p} \leq\left(\left|s_{i}\right|+\frac{1}{n}\right)^{p} \leq c_{p}\left(\left|s_{i}\right|^{p}+\frac{1}{n^{p}}\right) \\
& \sum_{i=1}^{\infty}\left|s_{i}-\frac{1}{n}\right|^{p} \leq \sum_{i=1}^{\infty} c_{p}\left(\left|s_{i}\right|^{p}+\frac{1}{n^{p}}\right) \leq c_{p} \sum_{i=1}^{\infty}\left|s_{i}\right|^{p}+c_{p} \sum_{i=1}^{\infty} \frac{1}{n^{p}}
\end{aligned}
$$

Como $\sum_{i=1}^{\infty}\left|s_{i}\right|^{p}$ converge para $s \in S_{\infty}$ y $\sum_{i=1}^{\infty} \frac{1}{n^{p}}$ es convergente para $p>1$, por el

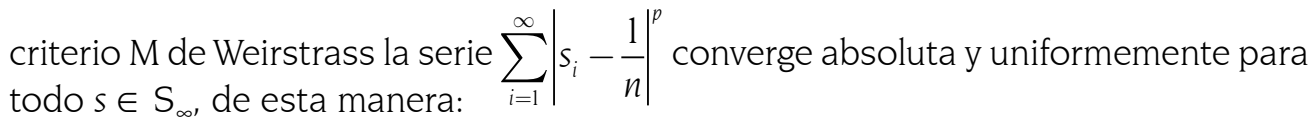

$$
\lim _{n \rightarrow \infty} \sum_{i=1}^{n}\left|s_{i}-\frac{1}{n}\right|^{p}=\sum_{i=1}^{\infty} \lim _{n \rightarrow \infty}\left|s_{i}-\frac{1}{n}\right|^{p}=\sum_{i=1}^{\infty}\left|s_{i}\right|^{p}
$$

Así, por continuidad de la potencia tenemos que:

$$
\mathrm{I}_{p, \infty}(s)=\lim _{n \rightarrow \infty}\left(\left.\sum_{i=1}^{n}\left|s_{i}-\frac{1}{n}\right|^{p}\right|^{\frac{1}{p}}=\left(\left.\lim _{n \rightarrow \infty} \sum_{i=1}^{n}\left|s_{i}-\frac{1}{n}\right|^{p}\right|^{\frac{1}{p}}=\left(\sum_{i=1}^{\infty}\left|s_{i}\right|^{p}\right)^{\frac{1}{p}}\right.\right.
$$

De esta manera, en el caso particular $p=2$ y una industria de $n$ firmas donde la participación industrial es $s=\left(s_{1}, s_{2}, \ldots, s_{n}\right)$ tenemos la siguiente relación con el índice de Herfindahl-Hirschman, es decir,

$$
\mathrm{I}_{2, \infty}(j(s))=\sqrt{\mathrm{IHH}(s)}
$$

O bien,

$$
\operatorname{IHH}(s)=\left(\mathrm{I}_{2, \infty}(j(s))\right)^{2}
$$

Donde $j$ es la inmersión de $\mathrm{S}_{n}$ en $\mathrm{S}_{\infty}$.

Así que hemos conseguido otra forma analítica de llegar al índice de HerfindahlHirschman a partir de límites de índices generados por normas, además dado que 
las normas lp son una generalización de las norma Euclídea, se puede pensar en los índices $\mathrm{I}_{p, \infty}$ como generalización de los índices de Herfindahl-Hirschman; esta generalización básicamente consiste en la generalización hecha por Hannah and kay los cuales son dados por

$$
\mathrm{HK}_{\alpha}(s)=\left(\sum_{i=1}^{n} s_{i}^{\alpha}\right)^{\frac{1}{\alpha-1}}
$$

Donde $\alpha>0$ y $\alpha \neq 1$ ( $\alpha$ es llamado el ponderador), y análogo al índice de Herfindahl-Hirschman, se presenta la siguiente relación para una industria de $n$ firmas donde la participación industrial es $s=\left(s_{1}, s_{2}, \ldots, s_{n}\right)$ con los índices $I_{p, \infty}$ cuando $p=\alpha$,

$$
\left(\mathrm{I}_{\alpha, \infty}(j(s))\right)^{\alpha}=\left(\mathrm{HK}_{\alpha}(s)\right)^{\alpha-1}
$$

Reescrito mejor como:

$$
\mathrm{HK}_{\alpha}(s)=\left(\mathrm{I}_{\alpha, \infty}(j(s))\right)^{\frac{\alpha}{\alpha-1}}
$$

Sin embargo, esta relación se cumple para valores de $\alpha>1$, ya que $p>1$ en las normas $1 p$, (notemos que para $0<p<1,\left(\sum_{i=1}^{n}\left|x_{i}\right|^{p}\right)^{\frac{1}{p}}$ no define una norma ya que
la bola unitaria no es convexa).

Al hacer un razonamiento idéntico para el índice $I_{\infty}$, definimos para cada $s \in$ $\mathrm{S}_{\infty}$ la función:

$$
\mathrm{I}_{\infty, \infty}(s)=\lim _{n \rightarrow \infty} n \frac{\max _{1 \leq i \leq n}\left|s_{i}-\frac{1}{n}\right|}{n-1}
$$

De igual forma $\lim _{n \rightarrow \infty} \frac{n}{n-1}=1$ y solo nos debemos ocupar de:

$$
\lim _{n \rightarrow \infty} \max _{1 \leq i \leq n}\left|s_{i}-\frac{1}{n}\right|
$$

Como $s \in \mathrm{S}_{\infty}$, existe un $\mathrm{N}>1$ tal que $s_{i}=0$ para $i \geq \mathrm{N}$, asi: 


$$
\max _{1 \leq i \leq n}\left|s_{i}-\frac{1}{n}\right|=\max \left\{\max _{1 \leq i \leq \mathrm{N}}\left|s_{i}-\frac{1}{n}\right|, \frac{1}{n}\right\}
$$

Así,

$$
\lim _{n \rightarrow \infty} \max _{1 \leq i \leq n}\left|s_{i}-\frac{1}{n}\right|=\max \left\{\lim _{n \rightarrow \infty} \max _{1 \leq i \leq \mathrm{N}}\left|s_{i}-\frac{1}{n}\right|, \lim _{n \rightarrow \infty} \frac{1}{n}\right\}=\max _{1 \leq i \leq \mathrm{N}} s_{i}=\max _{1 \leq i} s_{i}
$$

Por lo tanto, tenemos:

$$
\mathrm{I}_{\infty, \infty}(\mathrm{s})=\max _{1 \leq i} \mathrm{~s}_{i}
$$

Este índice se basa únicamente en la cuota de mercado que corresponde a la firma más dominante de la industria; esto nos hace recordar los índices más antiguos y usados como lo es el índice de concentración; este índice se define como la suma de las cuotas de mercado de las firmas más grandes, es decir, sea $s=\left(s_{1}, s_{2}\right.$, $\ldots, s_{n}$ ) una participación industrial ordenada de mayor a menor, se define el índice de concentración de orden $k$ por

$$
\mathrm{CR}_{k}(s)=\sum_{i=1}^{k} s_{i}
$$

En particular si $k=1$,

$$
\mathrm{CR}_{1}(s)=\max _{1 \leq i \leq n} \mathrm{~s}_{i}
$$

Así tenemos la relación,

$$
\mathrm{CR}_{1}(s)=\mathrm{I}_{\infty, \infty}(j(s))
$$

Para cualquier $s \in \mathrm{S}_{n}$.

\section{APLICACIÓN, VENTAJAS Y ANÁLISIS ECONÓMICO DE LOS ÍNDICES GENERADOS POR NORMAS}

Los siguientes datos corresponden a las ventas del sector automotriz, en el segmento de taxis nuevos, en mayo de 2016 en Colombia FENALCO-ANDI (2016); en esta sección se aplicará cada uno de los índices tratados en este trabajo, se discutirán sus bondades y desventajas. 
Carlos Fabián Ruiz Paredes - Luis Eduardo Ruiz Paredes - Jorge Morales Paredes

Tabla 1. Ventas sector automotriz en Colombia 2016

\begin{tabular}{|lllll|}
\hline \multicolumn{1}{|c|}{ Marca } & Cantidad & \multicolumn{1}{c|}{ Participación } & \multicolumn{1}{c|}{ Clasificación } \\
\hline Chevrolet & 149 & 0,173659674 & Dominante \\
Renault & 48 & 0,055944056 & No-dominante \\
Kia & 323 & 0,376456876 & Dominante \\
Suzuki & 12 & 0,013986014 & No-dominante \\
Hyundai & 257 & 0,2995338 & Dominante \\
Jac & 18 & 0,020979021 & No-dominante \\
Chery & 5 & 0,005827506 & No-dominante \\
Great Wall & 6 & 0,006993007 & No-dominante \\
Faw & 20 & 0,023310023 & No-dominante \\
Brillance & 2 & 0,002331002 & No-dominante \\
Lifan & 2 & 0,002331002 & No-dominante \\
Skoda & 9 & 0,01048951 & No-dominante \\
Dflz & 1 & 0,001165501 & No-dominante \\
Byd & 3 & 0,003496503 & No-dominante \\
Geely & 3 & 0,003496503 & No-dominante \\
\hline
\end{tabular}

Fuente: Fenalco y Andi (2016, p. 8-10)

El cálculo de los índices utilizados en este trabajo se muestra en la siguiente tabla:

Tabla 2. Indicadores del sector automotriz en Colombia 2016

\begin{tabular}{|ll|}
\hline Índice & Valor \\
\hline $\mathrm{IHH}$ & 0,26613635 \\
$\mathrm{I}_{p}, p=3$ & 0,38280276 \\
$\mathrm{I}_{p}, p=1,5$ & 0,55765064 \\
$\mathrm{I}_{1}$ & 0,69605395 \\
$\mathrm{I}_{\infty}$ & 0,33191808 \\
$\mathrm{I}_{p, \infty} p=3$ & 0,4408256 \\
$\mathrm{I}_{p, \infty} p=1,5$ & 0,62284067 \\
$\mathrm{I}_{2, \infty}$ & 0,51588405 \\
$\mathrm{I}_{\infty, \infty}=\mathrm{RC}_{1}$ & 0,37645688 \\
\hline
\end{tabular}

Fuente: elaboración propia 
Como en este casoa participación de las diferentes firmas en el mercado es completamente conocida y no existe ausencia de datos, es posible calcular todos los índices aquí presentados, gracias a la relación entre ellos (sección 3); la información que cada uno de ellos proporciona es muy similar (excepto el índice $\mathrm{RC}_{1}$ el cual omite la mayor parte de la información de las firmas), salvo los valores propios de cada escala; por ejemplo, la concentración $I_{2, \infty}=0,51588405$ es la misma descrita por $\mathrm{IHH}=0,26613635$, esto se debe a la escala que cada índice maneja (uno es la raíz cuadrada del otro); hasta este punto los índices comparten básicamente la misma información en términos de medición de concentración industrial, y por tal razón sería básicamente lo mismo trabajar con uno que con otro; sin embargo, gracias a la propiedad del índice generado por la norma del taxista o Manhattan dada por la igualdad:

$$
\mathrm{I}_{1}(s)=\frac{n}{n-1} \sum_{i \in \mathrm{D}} s_{i}-\frac{|\mathrm{D}|}{n-1}
$$

Esto permite calcular el valor del índice el cual básicamente describe la misma información en términos de concentración industrial que los otros (salvo su propia escala); es posible calcular el valor del índice únicamente conociendo la participación en el mercado de las firmas dominantes; en el caso del ejemplo estas serían:

Tabla 3 Firmas dominantes del sector automotriz Colombia 2016

\begin{tabular}{|l|l|l|}
\hline \multicolumn{1}{|c|}{ Marca } & \multicolumn{1}{c|}{ Cantidad } & \multicolumn{1}{c|}{ Participación } \\
\hline Chevrolet & 149 & 0,17365967 \\
\hline Kia & 323 & 0,37645688 \\
\hline Hyundai & 257 & 0,2995338 \\
\hline
\end{tabular}

Nota: estas marcas son dominantes ya que su participación de mercado está por encima del promedio de la participación total.

Fuente: Fenalco y Andi (2016)

En cuyo caso es:

$$
I_{1}(s)=\frac{15}{14}[0,17365967+0,37645688+0,2995338]-\frac{3}{14}=0,69605395 \quad[100]
$$

Lo anterior representa la mayor consecuencia de este trabajo ya que se llega a una medida de concentración industrial igual de poderosa que el índice de Herfindahl-Hirschman sin la limitante de la falta de datos; solo basta con conocer los 
datos de las firmas que están por encima de la media del mercado y el número de firmas; esto reduce significativamente el trabajo y los costos en la toma de datos; otra ventaja adicional de dicho índice es el principio débil de transferencia, que permite identificar plenamente los monopolios de las empresas grandes en un sector, es decir, si se hace traspaso de participación de mercado entre dos firmas dominantes (y estas siguen siendo dominantes) el valor del índice no varía, esto muestra que la concentración industrial depende exclusivamente de la participación de mercado de las firmas dominantes.

\section{CONCLUSIONES}

A partir de los índices generados por normas, los cuales son calculados fijando el número de firmas de una industria, se pueden, por medio del proceso del límite cuando el número de firmas es muy grande, generar índices en los cuales se elimine la dependencia del número de firmas; estos son precisamente los índices $\mathrm{I}_{p, \infty}$ y $\mathrm{I}_{\infty, \infty}$ (lastimosamente en el caso del índice generado por la norma del taxista o Manhattan este proceso no conduce a resultados interesantes) los cuales tienen una relación estrecha con los índices de Hannah and Kay y el índice de concentración de orden 1, respectivamente.

La manera en la que se construyen estos índices da una forma alterna matemática de llegar a las índices de Hannah and Kay (y en particular al Índice de HerfindahlHirschman) por medio de nociones básicas de distancia en espacios de dimensión finita y límites, apartándose de las formas tradicionales de llegar a ellos por medio de conceptos económicos.

Desde una perspectiva económica, los diferentes índices inducidos por normas muestran información valiosa en términos de concentración industrial o de mercado; sus relaciones estrechas con índices tradicionales brindan confiabilidad en cuanto a su uso y permiten analizar y comprender las dinámicas del mercado desde el ámbito de la organización industrial.

Un aspecto a tener en cuenta en el diseño de los índices aquí propuestos radica en la disminución de costos para la recolección de la información, gran desafío en términos muestrales; esto debido a que gracias al índice inducido por la norma del taxista la concentración de mercado puede llegar a calcularse únicamente conociendo las cuotas de mercado de las firmas dominantes, y así, reducir costos en términos generales.

La variedad de los índices aquí presentados, sin lugar a duda, permite tener una idea más clara y amplia en cuanto a la dinámica del mercado; la información 
adicional brindada por ellos muestra su ventaja frente a los índices tradicionales, y las estrechas relaciones existentes permiten tener confiabilidad a la hora de su uso tanto académico como por autoridades de competencia.

\section{BIBLIOGRAFÍA}

Apostol, Tom (2001). Análisis matemático. Reverté: Barcelona, España, 590p.

Bikker, Jacob y Haaf, Katharina. (2002). Measures of competition and concentration in the Banking Industry: a Review of the Literature. En: Research Series Supervision, No. 27, 35p.

Davies, Stephen (1979). Choosing between Concentration Indices: The Iso-Concentration Curve. En: Economica, Vol. 46, No. 181, febrero, p. 67-75.

Etro, Federico (2007). Competition, Innovation, and Antitrust: A theory of market leaders and its policy implications. Springer: Berlin, Alemania, 284p.

FENALCO y ANDI -Federación Nacional de Comerciantes y Asociación Nacional de Industriales- (2016). Informe del sector automotor a mayo de 2016. Colombia, 22p.

Hall, Marshall y Tideman, Nicolaus (1967). Measures of concentration. En Journal of the american statistical association, Vol. 62, No. 317, marzo, p. 162-168.

Hannah, Leslie y Kay, John (1977). Concentration in modern industry: theory, measurement, and the U.K. experience. Macmillan: London, UK, 144p.

Herfindahl, Orris (1950). Concentration in the U. S. Steel Industry.Tesis para optar al título de Doctor en Economía, PhD Economics, Universidad de Columbia, Estados Unidos.

Horvath, Janos (1970). Suggestion for a Comprehensive Measure of Concentration. En Southern Economic Journal, Vol. 36, No. 4, abril, p. 446-452.

Kreyszig, Erwin (1989). Introductory Functional Analysis with Applications. Wile: Estados Unidos, 704p.

Naldi, Maurizio y Flamini, Marta (2014). Correlation and Concordance between the CR4 Index and the Herfindahl-Hirschman Index. Social Science Research Network, 19p.

Ruiz Paredes, Carlos Fabian (2016). Índices de concentración industrial inducidos por métricas. Tesis para optar al título de Magister en Economía, Maestría en Economía, Universidad Externado de Colombia, Colombia, 74p. 